\title{
Universal reversal for heparin
}

\section{By Kai-Jye Lou, Senior Writer}

Titrating heparin treatment is a balancing act for hematologists trying to control blood coagulation without causing excessive bleeding. A synthetic heparin reversal agent from The University of British Columbia uses a dendritic polymer to neutralize the anticoagulant and could provide a lower-risk option for preventing bleeding during surgery than protamine, the standard of care. ${ }^{1}$ The challenge will be finding an industry partner to help move the candidate into clinical trials.

Protamine is the only approved antidote for heparin-based therapies. The cationic peptide works by binding to heparins, which are negatively charged, to form a complex that lacks anticoagulant activity. However, protamine has a narrow therapeutic window, has an unpredictable dose response and can cause significant adverse effects including a sudden drop in peripheral blood pressure, pulmonary hypertension and bradycardia.

"Protamine is a drug that has needed a replacement for a very long time," said James Costin, CMO and VP of clinical and medical affairs at Perosphere Inc. "Anything that can induce reversal of heparin anticoagulants without the liabilities of protamine would be very welcome, especially to cardiovascular surgeons."

Protamine's main activity is against unfractionated heparin (UFH), whereas it is only partially effective against low molecular weight heparin (LMWH) therapies and not effective against the generic fondaparinux, a synthetic ultralow molecular weight heparin (ULMWH).

LMWHs account for the lion's share of the heparin anticoagulants market, taking in $\$ 6.5$ billion in global sales last year. ${ }^{2,3}$ In contrast, global sales of UFH and fondaparinux are each well under $\$ 1$ billion.

"The strength of protamine's binding interaction is not sufficient to neutralize fondaparinux, and the biocompatibility of protamine and previously reported heparin-binding molecules has not been great," said Jayachandran Kizhakkedathu, an associate professor in the Department of Pathology and Laboratory Medicine at UBC. "What we want is something that binds heparin with high affinity that also has high biocompatibility."

To find a compound that could satisfy both criteria, the group created a series of heparin reversal agents based on a common structure containing three components: a dendritic polyglycerol core, trivalent cationic heparin-binding groups and short polyethylene glycol (PEG) chains (see Figure 1, "Structure of synthetic UHRAs").
Because heparins vary in size and are among the most highly negatively charged biological molecules, the goal was to produce compounds with optimally spaced cationic groups that could neutralize heparin-based anticoagulants universally but avoid binding common negatively charged molecules or surfaces including proteins, nucleic acids and cell membranes. The dendritic core of polyglycerol provides a structure for presenting multiple heparin-binding groups, and the PEG chains form a highly hydrated shell that protects the molecule from nonspecific interactions.

The team varied the size of the polymer core and the number of heparin-binding groups to optimize the molecular weight, charge distribution and density of the PEG shell and thereby maximize binding affinity and minimize off-target effects.

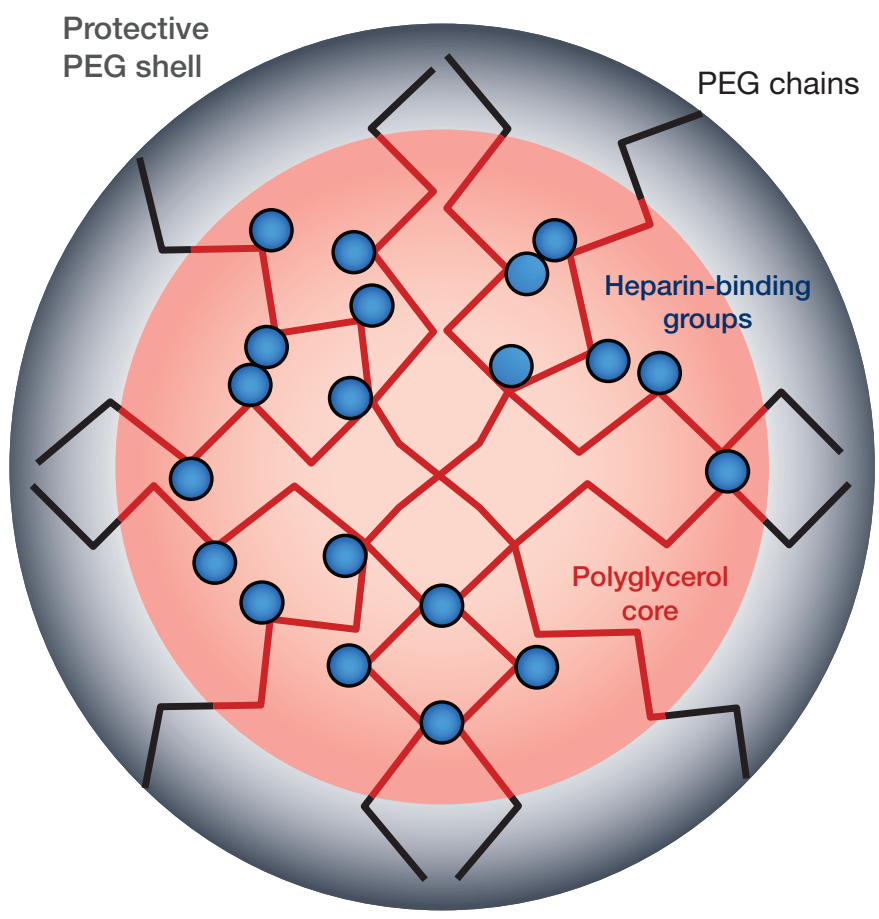

Figure 1. Structure of synthetic UHRAs. Researchers from The University of British Columbia have developed synthetic dendrimer polymers as universal heparin reversal agents (UHRAs) that could offer better safety profiles and biocompatibility than the standard of care, protamine.

The structure consists of a hyperbranched polyglycerol core (red lines) decorated with multiple cationic heparin-binding groups (blue circles). The polyglycerol branches are capped with short polyethylene glycol (PEG) chains (black lines) that form a protective shell (gray shading). The density of the PEG shell is optimized to enhance the biocompatibility of the overall structure and minimize its nonspecific interactions while preserving its binding affinity to heparins.

The lead agent described in Shenoi et al., UHRA-7, is decorated with 20 heparin-binding groups. ${ }^{1}$ 
In in vitro coagulation assays, various doses of the lead universal heparin reversal agent (UHRA), UHRA-7, caused complete or nearcomplete neutralization of UFH, the two tested LMWH therapies and fondaparinux. Although protamine also completely neutralized UFH, it was less effective than UHRA-7 at neutralizing the LMWHs and fondaparinux.

In vitro assays to measure aberrant blood coagulation, complement activation, platelet activation and red blood cell hemolysis suggested UHRA-7's biocompatibility is superior to that of protamine.

In rats, UHRA-7 completely neutralized UFH and the generic LMWH therapy enoxaparin. In contrast, although protamine completely neutralized UFH, it reversed enoxaparin activity by only about $60 \%$. In mice treated with UFH, fondaparinux or two different LMHW therapies, UHRA-7 decreased bleeding times and hemoglobin loss compared with the heparin therapy alone.

In mice, the highest tested dose of UHRA$7-50 \mathrm{mg} / \mathrm{kg}$ - showed no detectable toxicity, whereas the maximum tolerated dose for protamine was $20 \mathrm{mg} / \mathrm{kg}$ and a $30 \mathrm{mg} / \mathrm{kg}$ dose was lethal.

Results were published in Science Translational Medicine.

"The availability of this kind of universal heparin antidote would allow clinicians to think of new application settings for heparin therapies," said Kizhakkedathu. He noted that even though LMWH therapies and fondaparinux have better therapeutic profiles than UFH, their use in treating thrombotic events or cardiopulmonary bypass surgery has been limited in part by the lack of an effective antidote.

"We show that UHRA-7 works across a range of heparins and that it has a huge therapeutic window" compared with protamine, said Kizhakkedathu. He added that his group has since tested UHRA-7 at doses up to $300 \mathrm{mg} / \mathrm{kg}$ and has yet to reach the maximum tolerated dose.

Kizhakkedathu also noted that the synthesis process for the reversal agents is both straightforward and scalable and that the activity of the product across different fractions appears to be "very homogenous."

The UBC group is now improving the efficacy of its candidate compounds and assessing their potential for toxicity. The short-term plan is to test the compounds in preclinical bypass surgery models and in large animals. In addition, the researchers plan to prove that the synthesis process yields a uniform product and show that different fractions of the generated product retain the same level of activity. The immunogenicity of the agents also needs to be assessed.

Costin said that toxicity studies for the reversal agents should be carried out in a manner that assesses test compounds both alone and in combination with heparin.

"This is because the complex that forms between the reversal agent and the heparin drug is essentially a new molecule, so its toxicity will need to be assessed," he said. "They should also assess how well the reversal agent holds onto heparin to make sure there isn't some kind of rebound anticoagulant effect later on." According to Costin, even when protamine achieves successful heparin reversal, the protamine-heparin complex can uncouple at a later time point, resulting in a rebound anticoagulant effect.

\section{The path forward}

Steve Schow, industry consultant and former VP of R\&D at Telik Inc., told SciBX, "This is a particularly interesting approach in that the dendrimer is the drug, not a carrier of the drug or an excipient for the drug." He added that although there is activity in the field of dendrimer chemistry, if the compound makes it to market, it could become the first drug based on a dendritic scaffold.

MabVax Therapeutics Inc. reverse merged with Telik earlier this year to form MabVax Therapeutics Holdings Inc.

According to Kizhakkedathu, a likely clinical path forward for a lead compound would be to test it as a replacement for protamine in patients undergoing coronary artery bypass surgery. After that, he said, the compound could be tested for prevention of heparin-related bleeding complications in other settings.

Costin agreed that the bypass surgery setting is a good choice for initial development of the synthetic reversal agents. He said that subsequent usage settings to consider include procedures in catheterization laboratories or experiments involving cardiac electrophysiology.

However, moving ahead will likely require an industry partner, and Kizhakkedathu was cautiously optimistic about the prospects. $\mathrm{He}$ said that drug developers might view the market for antidotes against specific therapies as too small to justify the risk. However, he added that because his synthetic reversal agents appear to work against all heparin therapies, the potential market would likely be far larger than that for compounds that only work for a specific heparin therapy.

But the commercial landscape is not completely dry for heparin reversal. At least two companies-Portola Pharmaceuticals Inc. and Perosphere-are working on antidotes to heparin anticoagulants.

In June, Portola reported positive Phase II data showing that andexanet alfa could reverse the anticoagulant activity of enoxaparin. Andexanet alfa is a recombinant protein designed to sequester and neutralize factor $\mathrm{Xa}$ inhibitor-based anticoagulants. However, the recombinant protein also can neutralize heparin anticoagulants by binding to complexes of heparin and antithrombin III (AT3; SERPINC1) to prevent them from activating factor Xa.

In October, Portola said that andexanet alfa met the primary and secondary endpoints in the Phase III ANNEXA-A (Andexanet alfa, a Novel aNtidote to the anticoagulant Effects of fXA inhibitorsApixaban) trial of reversing the anticoagulant activity of Eliquis apixaban, a small molecule factor Xa inhibitor from BristolMyers Squibb Co. and Pfizer Inc. Andexanet has breakthrough designation from the FDA to reverse the anticoagulant activity of factor Xa inhibitors. The biotech plans to submit a BLA in late 2015. Portola declined requests for comment.

Kizhakkedathu noted that because his group's compounds are fully synthetic molecules, their immunogenicity risk and R\&D costs should be lower than those of protein-based antidotes such as andexanet alfa. However, he also noted that the agents are specific for reversing heparin anticoagulants and are unlikely to neutralize direct factor Xa inhibitors. 


\section{"The availability of this kind of universal heparin antidote would allow clinicians to think of new application settings for heparin therapies." \\ - Jayachandran Kizhakkedathu, The University of British Columbia}

neutralize its anticoagulant activity. PER977 also binds factor Xa inhibitor anticoagulants.

PER977 is in Phase II testing to reverse the anticoagulant activity of Daiichi Sankyo Co. Ltd.'s oral factor Xa inhibitor Lixiana edoxaban. The anticoagulant is approved in Japan and is under FDA review in the U.S., where it is called Savaysa.

Kizhakkedathu told SciBX that he is seeking funding or industry partners to carry out GMP manufacturing and GLP toxicity studies for advancing a lead universal heparin reversal agent to clinical trials.
Perosphere's a $\mathrm{nt}$ i coagulant antidote is PER977, a cationic synthetic small molecule that binds heparin via noncovalent hydrogen bonding and charge-charge interactions to a

\section{$\mathrm{CO}$}

CC

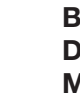

The University of British Columbia has filed a patent application covering polymers for reversing heparin-based anticoagulation. The technology is available for licensing.

Lou, K.-J. SciBX 7(47); doi:10.1038/scibx.2014.1366

Published online Dec. 11, 2014

REFERENCES

1. Shenoi, R.A. et al. Sci. Transl. Med.; published online Oct. 29, 2014; doi:10.1126/scitransImed.3009427

Contact: Jayachandran N. Kizhakkedathu, The University of British Columbia, Vancouver, British Columbia, Canada e-mail: jay@pathology.ubc.ca

2. Melnikova, I. Nat. Rev. Drug Discov. 8, 353-354 (2009)

. Chaudhari, K. et al. Nat. Rev. Drug Discov. 13, 571-572 (2014)

OMPANIES AND INSTITUTIONS MENTIONED

Bristol-Myers Squibb Co. (NYSE:BMY), New York, N.Y.

Daiichi Sankyo Co. Ltd. (Tokyo:4568) Tokyo, Japan

MabVax Therapeutics Holdings Inc. (OTCQB:MBVX), San Diego, Calif.

Perosphere Inc., Bedford, N.Y.

Pfizer Inc. (NYSE:PFE) New York, N.Y.

Portola Pharmaceuticals Inc. (NASDAQ:PTLA), South

San Francisco, Calif.

The University of British Columbia, Vancouver, British Columbia, Canada 